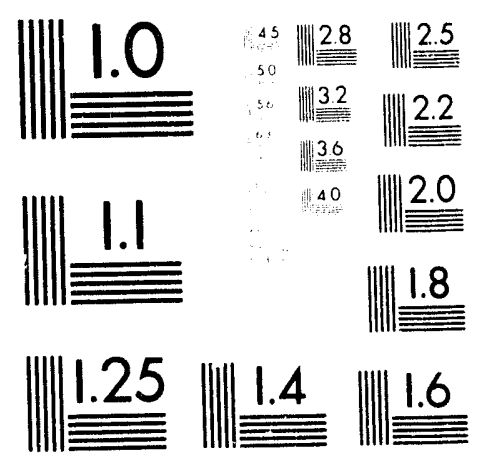



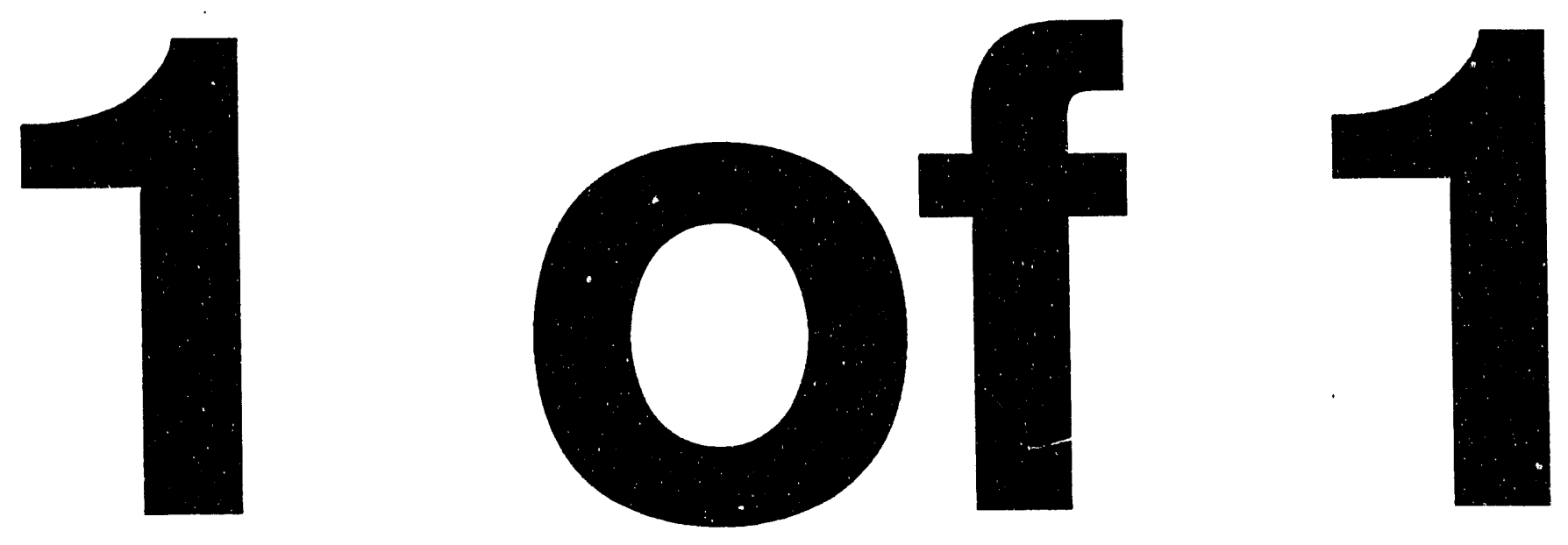


\title{
SIMPLE ESTIMATE OF STORED ENERGY AND CONDUCTOR INSULATION VOLTAGE-WITHSTAND REQUIREMENTS FOR AD VANCED SOLENOID MAGNET DESIGNS *
}

\author{
S. W. Schwenterly and L. Dresner \\ Oak Ridge National Laboratory \\ Oak Ridge, TN 37831
}

\begin{abstract}
Compact, lightweight, high-current-density superconducting magnets are of interest for many applications. Minimizing the area taken up by conductor stabilizer material and insulation can lead to high dump voltages if the magnet is protected by discharging it through an external resistor. The dump roltages per turn for circular solenoids are estimated for this situation, in terms of th: well-known "hotspot integral" $H(\Delta T)$, the maximum on-axis magnetic field $B_{z}$, and the: stored energy $E$. The field and stored energy are calculated in terms of the coil geometry parameters $\alpha$ and $\beta$. The results depend only on $B_{z}, H(\Delta T)$, and certain scaling factors, and are independent of the coil size. The relations give simple estimates of the average turn dump voltages for a coil of a given field and geometry which are useful for preliminary scoping studies during conceptual design of the magnet.
\end{abstract}

\section{INTRODUCTION}

Advances in superconductor design and development of new high-strength polymer matrix composite (PMC) materials point to the possibility of constructing very compact, lightweight magnets with high winding current densities and structural stresses. To reduce weight and volume, overall current densities of 10 to $20 \mathrm{kA} / \mathrm{cm}^{2}$ are desirable. Further development of the high-temperature superconductors (HTS) will hopefully lead to magnets which can operate at much higher fields and temperatures than previously possible. Applications for these magnets include magnetic levitation, ship propulsion, energy storage, motors, and magnetic resonance imaging. Since many of the advanced structural composites are electrical insulators, or at least semiconductors, these structural materials might also serve as electrical insulation in the magnet winding. The magnet insulation must withstand voltages between turns and between layers $\mathrm{cr}$ pancakes, as well as the total voltage developed between leads or from the leads to ground.

The highest voltages will generally occur when the magnet energy is dumped quickly into an external resistor in the event of a normal zone. This process is particularly necessary for HTS conductors, which display very slow normal zone propagation rates 1 and thus cannot be allowed to dissipate the entire stored energy within the winding. The dump voltage is distributed in various fractions among the individual trens and among the

* Research supported by U.S. D.O.E. Superconducting Technology Program, B\&R\# AK 0600000 under contract \# DE-AC05-84OR21400 with Martin Marietta Energy Systems, Inc. 
layers or pancakes of the winding. This paper summarizes some calculations we have made to estimate the turn voltages which could appear between turns and between layers or pancakes for magnets of various bore sizes, geometries, and fields.

\section{CALCULATION OF DUMP VOLTAGE}

If the magnet is discharged through a dump resistor which is large compared to the normal zone resistance, the dump voltage per turn required to keep its final temperature below some desired value $T_{f}$ is given by

$$
\frac{V_{d}}{N}=\frac{[N I E]}{A^{2} H(\Delta T)}
$$

A derivation of this relation can be found in Reference 2. A.ll variables are defined in a table at the end of the paper. MKS units are assumed, with magnetic fields in $T$. It is assumed that heat transfer out of the normal zone is neglible and that all current transfers to the stabilizer, in order to give an estimate of the maximum heating. It can be argued that this is somewhat unrealistic for HTS since it can carry some current up to temperatures approaching $100 \mathrm{~K}$, but the heat capacity is so high that the only credible event which could cause current transfer is a wholesale mechanical failure which destroys the HTS material. The "hotspot integral" $\mathrm{H}(\Delta T)$ is given by

$$
H(\Delta T)=\int_{T_{i}}^{T_{f}} \frac{S(T)}{\rho(T)} d T
$$

where $T_{i}$ and $T_{f}$ are the initial and final temperatures. The conductor heat capacity $S(T)$ includes any structural material in contact with the conductor. $H(\Delta T)$ depends on the conductor configuration, allowed temperature rise, and magnetic field. Tables or plots of $H(\Delta T)$ are readily available in the literature for conventional low-temperature supe conductors (LTS)- - see, for example, Reference 3. For NbTi at $4.2 \mathrm{~K}$ with a final temperature of $100 \mathrm{~K}$, a field of $8 \mathrm{~T}$, and a copper:SC ratio of 4.4 , we estimate its value as

$$
H(\Delta T)=7.0 \times 10^{16} \mathrm{~A}^{2}-\mathrm{m}^{-4}-\mathrm{sec} .
$$

For HTS materials, the favored stabilizer is silver. Fig. 1 shows plots of $H(\Delta T)^{1}$ for silver at various resistivity ratios. For BSCCO HTS material, this function can be corrected for the Ag:BSCCO fraction by a simple multiplicative factor 1 , which is about 0.85 for an Ag:BSCCO ratio of 4.4. The RRR $=500$ curve is typical of currently-produced material. Thus, for $T_{i}$ of $40 \mathrm{~K}$ and $T_{f}$ of $100 \mathrm{~K}$, we estimate for HTS

$$
H(\Delta T)=3.0 \times 10^{10} \mathrm{~A}^{2}-\mathrm{m}^{-4}-\mathrm{sec} .
$$

Let us consider a solenoid coil with an inner bore radius of $a_{1}$, an outer radius of $a_{2}$, and half-length $b$. If all the dimensions of the coil are given in terms of $a_{1}$, then the stored energy can be expressed by

$$
E=1 / 2 L I^{2}=\varepsilon a_{1}(N I)^{2}
$$

Here $\varepsilon$ is a geometric coefficient which is the same for all coils of given relative proportions, no matter what their actual size. It is a function of $\alpha$, the ratio of $a_{2}$ to $a_{1}$, and $\beta$, the ratio of $b$ to $a_{1}$. It can be calculated from coil inductance formulas and tables such as those given by Grover ${ }^{4}$. However, for the present paper, a computer calculation of the inductance using the Fawzi-Burke method 5 was used. Fig. 2 shows the inductance of a 1$\mathrm{m}$-bore-radius, one-turn coil (i.e., $2 \varepsilon$ ) as a function of $\beta$, for values of $\alpha$ ranging from 1.02 to 3 . 


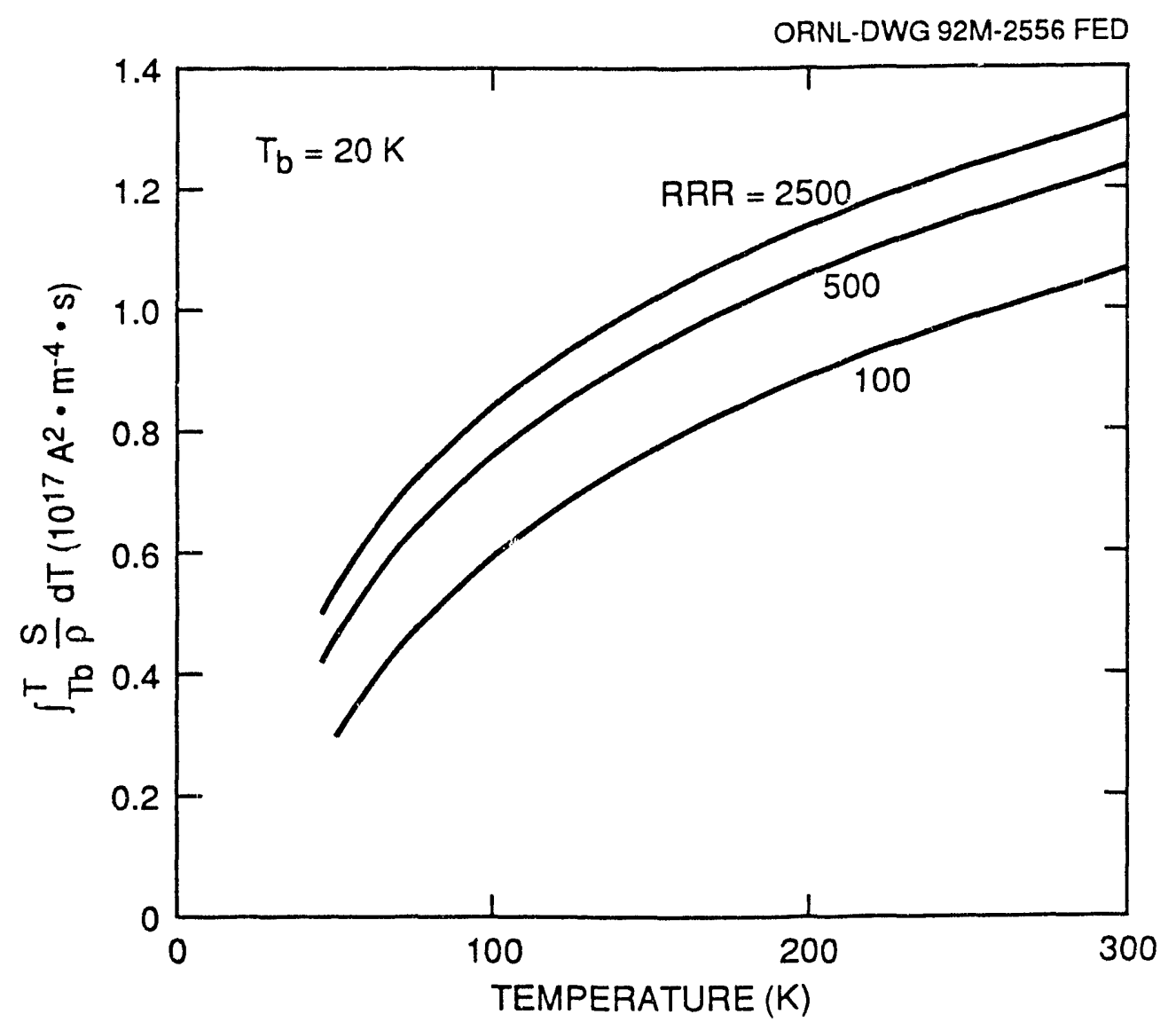

Figure 1. Hotspot integral for silver vs temperature for various values of residual resistivity.

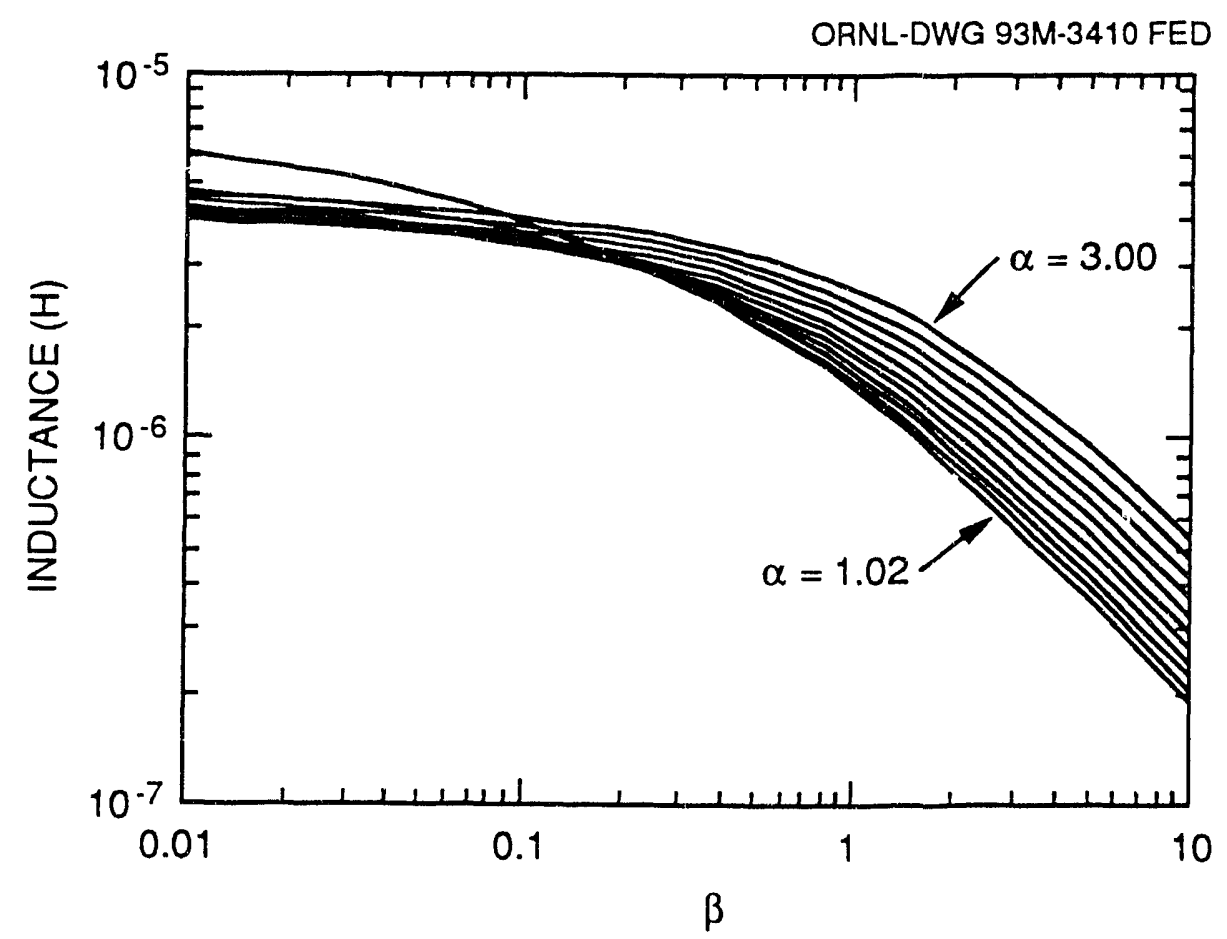

Figure 2. Inductance of a one-turn coil with 1-m radius vs $\beta$ at various values of $\alpha$. Each successive value of $\alpha$ is a factor of 1.114 higher than the previous value. 
The amp-turns $N I$ are related to the maximum magnetic field $B_{z}$ on axis at the center of the coil by

$$
N I=\phi a_{1} B_{z}
$$

Here $\phi$ is another geometric coefficient related to the coil proportions. This is basically a consequence of Ampere's Law. $B_{z}$ and thus $\phi$ can be easily calculated from the relation 6

$$
B_{z}=\left[\frac{\mu_{0}}{2(\alpha-1)}\right] \ln \left[\frac{\alpha+\sqrt{\alpha^{2}+\beta^{2}}}{1+\sqrt{1+\beta^{2}}}\right] \frac{N I}{a_{I}}
$$

If it is desired to characterize the coil by the field at some off-axis point (such as the maximum field at the coil windings), then the field at this point is most easily determined from a magnetic field calculation code.

The total stabilizer area $A$ in the winding pack is determined by the coil geometry and stabilizer fraction $f$. This factor includes turn insulation, stabilizer: $S C$ ratio, coolant volume, and any structural sheath around the conductor. Thus, if all dimensions are defined in terms of the bore radius $a_{1}$,

$$
A=2 f(\alpha-1) \beta a_{1}^{2}=\gamma_{a_{1}}^{2}
$$

where $\gamma$ is another coefficient which takes into account all the above factors.

Substituting (3), (4), and (6) into (1), the dump voltage per turn is

$$
\frac{V_{d}}{N}=\frac{\varepsilon \phi^{3}\left(B_{z}\right)^{3}}{\gamma^{2} H(\Delta T)}
$$

Thus the dump voltage per turn is expressed in terms of parameters to be chosen by the designer. It is interesting that when expressed in terms of the magnetic field, the voltage is independent of the coil dimension and number of turns, once the geometry has been chosen. The voltage is very sensitive to the choice of the characteristic magnetic field, and can vary considerably in a geometry where the field is not uniform. Note that this is an average voltage per turn, which does not consider capacitances between layers or coupling with any conductive casing around the winding. If the coil has many turns or a high ground capacitance compared to the series capacitance, the initial voltage distribution can be very non-linear. It is possible for the maximum turn voltage to exceed the average value by an order of magnitude 7 . Internal voltage oscillations inside the coil during the dump transient can also cause the total voltage to reach $150 \%$ of the initia! value 7,8 . These effects can be determined only after a detailed coil design has been chosen.

The overall current density in the winding is the ratio $f N I / A$. However, the current density cannot be chosen independently of the geometry and magnetic field without affecting the dump voltage per turn, because $N I$ is determined by (4), and $A$ is determined by (6). It may be necessary to iterate the calculation with a modified winding design to reach a desired overall current density. The number of turns in the winding pack can then be chosen to yield a convenient conductor current and/or total dump voltage.

\section{EXAMPLES}

\section{Brooks Coil}

Consider first a Brooks coil, which has inner and outer radii of $a_{1}$ and $2 a_{1}$, and a length of $a_{1}$. Thus the winding pack is a $a_{1} \times a_{1}$ square. This geometry produces the maximum inductance for a given length of conductor. Calculations of the stored energy and magnetic field with $\alpha=2$ and $\beta=0.5$ give 


$$
E=\left(1.281 \times 10^{-6}\right) a_{1}(N)^{2} \text { and } N I=\left(2.444 \times 10^{6}\right) a_{1} B_{z}
$$

Suppose that $B_{z}$ is $8 \mathrm{~T}$, and $H(\Delta T)$ is as given above for LTS and HTS. If the winding pack is $60 \%$ stabilizer, we have $A=(0.6) a_{1}^{2}$, and so

$$
V_{d} / N=0.38 \mathrm{~V} / \text { turn for LTS, and } 0.89 \mathrm{~V} / \text { turn for HTS. }
$$

Thus the voltage across two layers with say, 100 turns each would be about $76 \mathrm{~V}$. If the whole winding contains 10,000 turns, the total voltage would be $3800 \mathrm{~V}$. If we take $a_{1}=0.5 \mathrm{~m}$, the overall winding current density would be $3.9 \mathrm{kA} / \mathrm{cm}^{2}$. The conductor current would be $978 \mathrm{~A}$.

\section{Long Solenoid}

Suppose now that we consider a solenoid with the same inner and outer radii as the Brooks coil, but ten times its length. In this case, with $\alpha=2$ and $\beta=5$,

$$
E=\left(3.122 \times 10^{-7}\right) a_{I}(N I)^{2} \text { and } N I=\left(8.318 \times 10^{6}\right) a_{l} B_{Z} .
$$

The total stabilizer area is $A=(6.0) a_{1} 2$. With the same values for the other parameters,

$$
V_{d} / N=0.037 \mathrm{~V} / \text { turn for LTS, and } 0.086 \mathrm{~V} / \text { turn for HTS. }
$$

The turn voltage is only $1 / 10$ that for the Brooks coil because this geometry does not store as much energy per unit volume of winding, due to the fact that the field is close to zero outside a long solenoid except near the ends. The overall current density would drop to $1.3 \mathrm{kA} / \mathrm{cm}^{2}$ for $a_{1}=0.5 \mathrm{~m}$.

\section{Pancake Coil:}

Consider now a pancake coil with an inner radius of $a_{1}$, an outer radius of $11 a_{1}$, and a length of $a_{1}$. Thus the winding cross section is the same as for the long solenoid coil. With $\alpha=11$ and $\beta=0.5$,

$$
E=\left(4.479 \times 10^{-6}\right) a_{1}(N)^{2} \text { and } N I=\left(6.798 \times 10^{6}\right) a_{1} B_{Z} .
$$

The total stabilizer area is again $A=(6.0) a_{1}{ }^{2}$. With the same values for the other parameters,

$$
V_{d} / N=0.29 \mathrm{~V} / \text { turn for LTS, and } 0.68 \mathrm{~V} / \text { turn for HTS. }
$$

Thus, with the same number of turns arranged in a pancake instead of a solenoid, the turn voltage again approaches the Brooks coil voltage. The overall current density required for $8 \mathrm{~T}$ and $a_{1}=0.5 \mathrm{~m}$ is about $1.1 \mathrm{kA} / \mathrm{cm}^{2}$.

\section{"Thin" Brooks Coil:}

Let us consider a coil with the same bore and length $a_{l}$ as a Brooks coil, but a winding thickness of only $1 / 4 a_{1}$. With $\alpha=1.25$ and $\beta=0.5$,

$$
E=\left(1.085 \times 10^{-6}\right) a_{1}(N I)^{2} \text { and } N I=\left(1.954 \times 10^{6}\right) a_{1} B_{z} .
$$

The total stabilizer area is $A=(0.15) a_{1}{ }^{2}$. With the same values for the other parameters,

$$
V_{d} / N=2.63 \mathrm{~V} / \text { turn for LTS, and } 6.14 \mathrm{~V} / \text { turn for HTS. }
$$

With $a_{1}=0.5 \mathrm{~m}$, the required overall current density works out to about $12.5 \mathrm{kA} / \mathrm{cm}^{2}$. Hence, sizeable turn voltages and current densities start developing when the winding 
thickness becomes small compared to the coil bore. A conductc current of about $5 \mathrm{kA}$ is reasonable for this size coil. Thus, the area per turn is about $0.4 \mathrm{~cm}^{2}$. A $52 \times 30$ winding pack with 1560 turns would fill the available space. If wound in layers, the layer voltage would be 137 volts, and the total dump voltage would be about $4100 \mathrm{~V}$ for LTS and $9580 \mathrm{~V}$ for HTS.

\section{Large Ring Coil:}

For comparison, let us look at a much larger ring coil such as might be used as a poloidal field coil in a fusion reactor. Suppose that the bore radius is $4.0 \mathrm{~m}$ and the winding pack is $1.0-\mathrm{m}$ square. For this coil with $\alpha=1.25$ and $\beta=0.125$,

$$
E=\left(1.700 \times 10^{-6}\right) a_{l}(N)^{2} \text { and } N I=\left(1.795 \times 10^{6}\right) a_{l} B_{z} .
$$

For such large ring coils, there is a large variation in field with radius, and they are usually rated by field at the conductor rather than at the center of the bore. For a coil with these proportions, the field at the center is only about a third of that at the conductor. Thus, for $8 \mathrm{~T}$ at the conductor, there is only $2.6 \mathrm{~T}$ at the bore center, and this is the appropriate value for $B_{2}$ in the above relation. The total stabilizer area is $A=(0.0188) a_{1} 2$. The area coefficient is quite small because the winding pack dimensions are much smaller than the bore. Also, we assume that the coil has only $30 \%$ stabilizer in the winding pack to allow for a structural sheath on the conductor. With these values, the overall current density is $2.1 \mathrm{kA} / \mathrm{cm}^{2}$, and

$$
V_{d} / N=6.48 \mathrm{~V} / \text { turn for LTS, and } 15.12 \mathrm{~V} / \text { turn for HTS. }
$$

\section{USE OF PMC FOR ELECTRICAL INSULATION}

Suppose that a preliminary design for the coil has been determined, and that the designer has decided that a certain thickness of PMC material is needed on the conductor to support the forces on it. When this material is also used for electrical insulation, current will flow through it during a coil dump if its resistivity is low. This will contribute additional heat to that generated in the conductor. A rough estimate of the extra heating can be made as follows.

If the dump voltage across the insulation next to the hot spot decays exponentially and $R_{i}$ is the insulation resistance to the neighboring turn for a $1-\mathrm{m}$ length of conductor, then the heat produced in this 1-m length during a dump by current flow through the insulation between turns is

$$
Q_{i}=\frac{\left(V_{d} / N\right)^{2}}{R_{i}} \int_{0}^{\infty} e^{-2 t / \tau} d t=\frac{\left(V_{d} / N\right)^{2} \tau}{2} \frac{.}{R_{i}}
$$

Since $\tau=L / R_{d}=2 E / I V_{d}$, this leads to

$$
Q_{i}=\frac{E}{N^{2} I R_{i}} V_{d}
$$

For both $\mathrm{Cu} / \mathrm{NbTi}$ and $\mathrm{Ag} / \mathrm{BSCCO}$, the effective heat capacity of the conductor at $100 \mathrm{~K}$ is about $S_{\text {eff }}=2 \mathrm{~J}-\mathrm{cm}^{-3}-\mathrm{K}^{-1}$, and is not increasing very rapidly with temperature. Then the temperature rise in the conductor due to resistance heating in the insulation is

$$
\Delta T_{r}=Q_{i} / S_{\text {eff }} v=\frac{E}{S_{\text {eff }} v N^{2} I R_{i}} V_{d}
$$

where $v$ is the volume of the conductor per $\mathrm{m}$. 
Consider the "thin" Brooks coil discussed previously. Suppose that there is highresistance insulation between layers, so that insulation current can flow only between neighboring turns. We had dump voltages of $4100 \mathrm{~V}$ and $9580 \mathrm{~V}$, a bore radius of $0.5 \mathrm{~m}$, 1560 turns, and $5 \mathrm{kA}$ conductor current. We have determined by experiment that the roomtemperature resistivity of a carbon fiber-epoxy composite with a thickness of $0.2 \mathrm{~mm}$ is about $1 \Omega-\mathrm{m}$. The $100-\mathrm{K}$ resistivity will probably be at most $50 \%$ higher, if the material behaves like a carbon resistor. The turn height is $0.004 \mathrm{~m}$, and so the resistance $R_{i}$ between turns of $1 \mathrm{~m}$ of such insulation is about $0.08 \Omega$. The above relation gives $\Delta T_{r}=2 \mathrm{~K}$ for LTS and $4.6 \mathrm{~K}$ for HTS. Thus, even a rather low insulation resistivity does not lead to very much extra heating. However, it must be kept in mind that during a discharge heat is generated in the insulation inroughout the coil, not just at the normal zone. This could lead to very high heat loads for coils which are discharged frequently. For such coils, an appropriate insulation resistance criterion might be that the average insulation power dissipation be no greater than the current lead heat load. For the "thin" Brooks coil, this would lead to required insulation resistivities greater than $10^{6} \Omega-\mathrm{m}$.

\section{CONCLUSION}

A method has been outlined for estimating required dump voltages of solenoid magnets from the field rating, coil proportions, and hotspot integral. The resulting turn voltages are independent of magnet size, and depend instead on the relative proportions of the winding pack. For solenoid magnets with a field rating of $8 \mathrm{~T}$, overall winding pack current densities near $10 \mathrm{kA} / \mathrm{cm}^{2}$ result in turn voltages in the range of a few volts for LTS and 10 volts for HTS. Much greater voltages can be generated by very large coils which require a high fraction of structural material in the winding pack.

Estimates of the heating in low-resistivity turn insulation during a single discharge indicate that the extra temperature rise is not excessive for resistivities as low as $1 \Omega$-m. For coils which discharge frequently, much higher resistivities are required. The resistivities of the advanced composites which appear most interesting for superconducting magnets need to be determined in the range from $4 \mathrm{~K}-150 \mathrm{~K}$ and compared with these values. Low-temperature heat capacity and thermal conductivity data would also be useful in order to assess thermal transients and heat transfer in the insulation.

\section{REFERENCES}

1. L. Dresner, Stability and Protection of Ag/BSCCO Magnets Operated in the 20-40 K Range, to be published in Cryogenics, September, 1993.

2. S. W. Schwenterly, Influence of Dump Voltage and Allowable Temperature Rise on Stabilizer Requirements in Superconducting Magnets, IEEE Trans. Magn. 25:1717 (1989).

3. M. N. Wilson, “Superconducting Magnets", Clarendon Press, Oxford (1983), pp. 201-202 .

4. F. W. Grover, "Inductance Calculations- Working Formulas and Tables," Dover, New York (1962).

5. T. H. Fawzi and P. E. Burke, The Accurate Computation of Self and Mutual Inductances of Circular Coils, IEEE Tr. Power App. Sys. PAS-97 (2):464 (1978).

6. D. B. Montgomery, "Solenoid Magnet Design,” Wiley, New York (1969).

7. H. Brechna, "Superconducting Magnet Systems," Springer, New York (1973), pp. 332-339.

8. P. Chowdhuri and M. Anderson, Performance of Large Magnets Under Transient Voltages, Proc. 9th Symposium on Eng. Probs. of Fusion Research, IEEE (1981), p. 448.

\section{DISCLAIMER}

\footnotetext{
This report was prepared as an account of work sponsored by an agency of the United States Government. Neither the United States (jovernment nor any agency thereof, nor any of their employees, makes any warranty, express or implied, or assumes any legal liability or responsibility for the accuracy, completeness, or usefulness of any information, apparatus, product, or process disclosed, or represents that its use would not infringe privately owned rights. Reference herein to any specific commercial product, process, or service hy trade name, trademark, manufacturer, or otheru ise does not necessarily constitute or imply its endorsement, recommendation. or favoring by the United States Government or any agency thereof. The views and opinions of authors expressed herein do not necessarily state or reflect those of the United States Government or any agency thereof.
} 


\section{LIST OF VARIABLES}

$\begin{array}{llll}A & \text { Total stabilizer area in winding pack } & \alpha & a_{2} / a_{1} \text { ratio } \\ a_{1}, a_{2} & \text { Inner and outer radius of coil } & \beta & b / a_{1} \text { ratio } \\ b & \text { Coil half-length } & \varepsilon & \text { Stored energy coefficient } \\ B_{z} & \text { Maximum magnetic field on axis } & \phi & \text { Magnetic field coefficient } \\ E & \text { Stored energy of coil } & \gamma & \text { Stabilizer area coefficient } \\ f & \text { Fraction of stabilizer in winding pack } & \rho(T) & \text { Stabilizer resistivity } \\ H(\Delta T) & \text { Hotspot Integral } & \tau & \text { Coil dump time constant } \\ I & \text { Conductor current } & \\ L & \text { Coil inductance } & \\ N & \text { Number of turns in coil } & \\ Q_{i} & \text { Joule heat generated in 1 m of turn electrical insulation } \\ R_{i} & \text { Insulation resistance of 1 m of turn electrical insulation } \\ S(T) & \text { Conductor heat capacity per unit volume } & \\ T_{f} & \text { Final hotspot temperature at dump } & \\ T_{i} & \text { Initial hotpot temperature at dump } & \\ V_{d} & \text { Dump voltage } & \\ v & \text { Volume or conductor per m } & \end{array}$



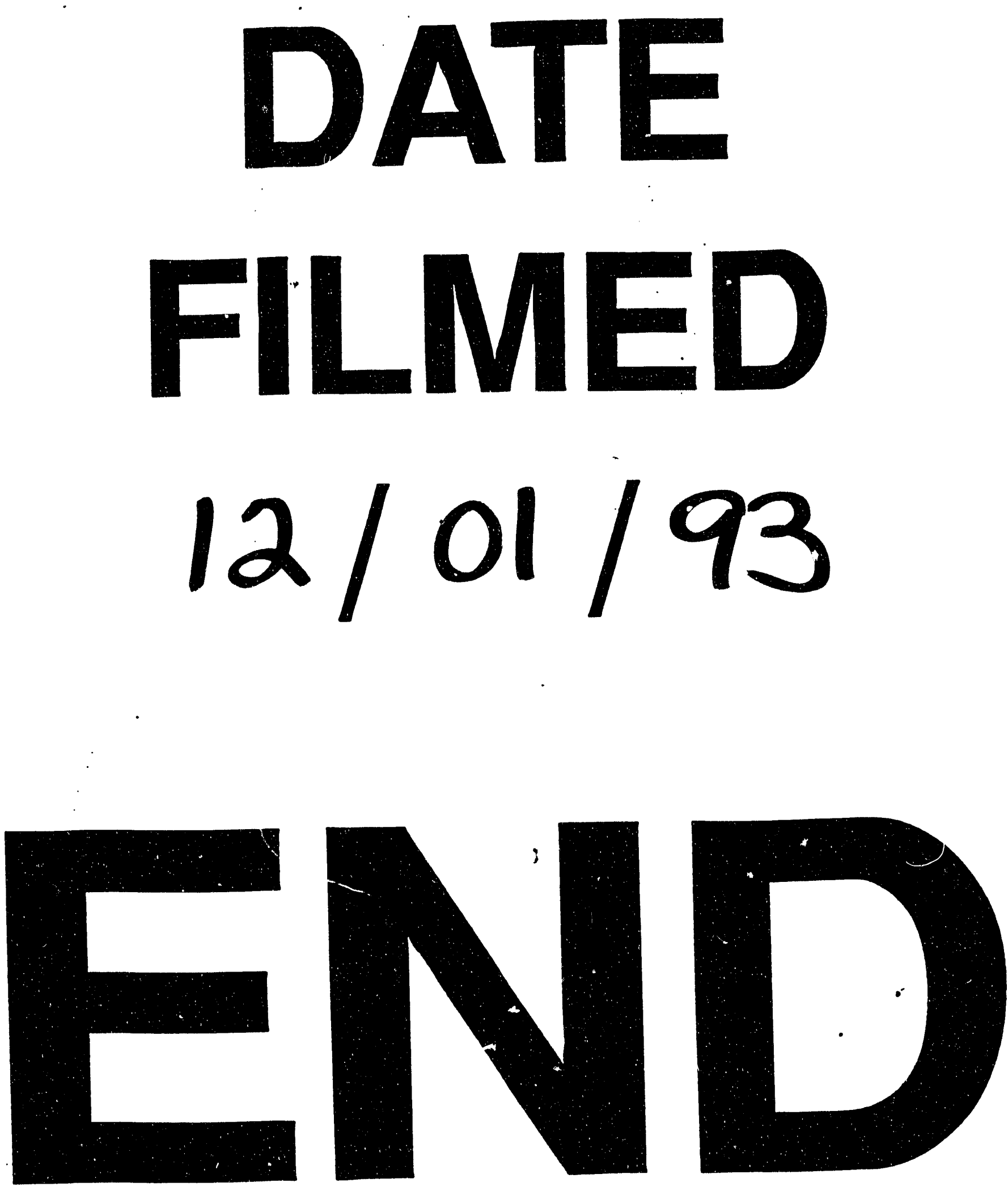
